\title{
Transvenous Aneurysm Sac and Rupture Point Coil Embolization of Direct Carotid Cavernous Fistula after Pipeline Embolization
}

\author{
Hidenori Oishi, ${ }^{1,2}$ Kosuke Teranishi, ${ }^{2}$ Kenji Yatomi, ${ }^{2}$ Munetaka Yamamoto, ${ }^{2}$ and Hajime Arai $^{2}$
}

\begin{abstract}
A delayed aneurysm rupture after flow diverter therapy is a rare but serious complication. Due to the anatomical specificity, a delayed rupture of a carotid cavernous aneurysm may cause a direct carotid cavernous fistula (dCCF). We present a novel therapeutic approach for treatment of dCCF after flow diverter therapy using the Pipeline embolization device (PED). An 86-year-old woman suffered from dCCF after PED embolization. A microcatheter was advanced through the transvenous approach into the cavernous sinus (CS) and further inserted into the aneurysm sac via the rupture point. Coil embolization of both the aneurysm sac and a small part of the CS adjacent to the fistulous site could achieve not only the immediate aneurysm occlusion but also the rupture point obliteration with a small amount of coil mass in the CS.
\end{abstract}

Keywords: transvenous coil embolization, flow diverter, carotid cavernous fistula, delayed aneurysm rupture, pipeline embolization device

\section{Introduction}

The Pipeline embolization device (PED; Medtronic Neurovascular, Irvine, CA, USA) is a popular flow diverter for the treatment of large or giant unruptured intracranial aneurysms. A delayed aneurysm rupture is one of the serious complications of the embolization procedure. ${ }^{1-6)}$ We report a case of direct carotid cavernous fistula (dCCF) after PED embolization of a carotid cavernous aneurysm treated with transvenous aneurysm sac and fistulous site coil embolization.

\section{Case Report}

An 86-year-old woman complained of double vision due to a right incomplete oculomotor nerve palsy. Magnetic resonance imaging showed a right carotid cavernous aneurysm (Fig. 1). She was nonsmoker, and had no history of hypertension. Digital subtraction angiography (DSA) images confirmed the aneurysm measuring $20 \mathrm{~mm}$ in diameter (Fig. 2). PED embolization was scheduled and the patient was given dual

${ }^{1}$ Department of Neuroendovascular Therapy, Juntendo University School of Medicine, Tokyo, Japan

${ }^{2}$ Department of Neurosurgery, Juntendo University School of Medicine, Tokyo, Japan

Received: April 28, 2017; Accepted: May 30, 2017

Online November 28, 2017

Copyright $\odot 2018$ by The Japan Neurosurgical Society

This work is licensed under a Creative Commons Attribution-

NonCommercial-NoDerivatives International License. antiplatelet therapy (DAPT) on aspirin $100 \mathrm{mg} / \mathrm{d}$ and clopidogrel $50 \mathrm{mg} / \mathrm{d} 10$ days prior to the procedure. Platelet activity measured by VerifyNow (Accumetrics, San Diego, CA, USA) assays within 12 hours before the intervention showed aspirin reaction units $460 \mathrm{P} 2 \mathrm{Y} 12$ reaction units 135 . Under general anesthesia and systemic heparinization, a 7 French (F) long sheath was placed in the right internal carotid artery (ICA) via femoral artery puncture. A Navien $5 \mathrm{~F}$ intracranial support catheter (Medtronic Neurovascular, Irvine, CA, USA) was placed in the petrous segment of the ICA and a Marksman microcatheter (Medtronic Neurovascular) was introduced into the right M1 segment with the assistance of a microguidewire. Three PEDs $(4.5 \mathrm{~mm} / 30 \mathrm{~mm}, 4.75 \mathrm{~mm} / 30 \mathrm{~mm}, 5 \mathrm{~mm} / 30 \mathrm{~mm})$ were telescopically deployed (Fig. 3). Posttreatment DSA images showed significant contrast stasis within the aneurysm (Fig. 4). The postoperative course was uneventful, and the patient was discharged at the neurological baseline 10 days after the procedure with the same DAPT regimen. Six weeks after the PED embolization, the patient suffered from sudden onset of a severe headache following pulsatile tinnitus and progressive worsening of the right visual acuity without any head injuries. DSA images showed the development of a right $\mathrm{dCCF}$ that drained into the right superior ophthalmic vein, the pterygoid venous plexus, the inferior petrosal sinus, the superior petrosal sinus, and via the intercavernous sinus into the contralateral cavernous sinus (CS) (Figs. 5A and 5B). Because we determined from the evaluation of a balloon occlusion test (BOT) that the patient could not tolerate a permanent carotid occlusion, a transvenous coil embolization was indicated as a treatment option to preserve the ICA flow. Under general anesthesia and systemic heparinization, a $4 \mathrm{~F}$ diagnostic catheter was inserted into the right ICA via the left femoral artery sheath to provide a roadmap guidance and control angiography. After the $6 \mathrm{~F}$ sheath was introduced in the right common femoral vein, the tip of the $4 \mathrm{~F}$ inner catheter coaxially assembled with the $6 \mathrm{~F}$ guiding catheter was cannulated into the origin of the right inferior petrosal sinus via the internal jugular vein. A microcatheter (Excelsior SL-10; Stryker Neurovascular, Fremont, CA, USA) was advanced into the CS and further inserted into the aneurysm sac via the rupture point with the assistance of the microguidewire (Fig. 6). After the aneurysm sac was embolized with some coils, the microcatheter was pulled back into the CS, and a few coils were put into the part of the CS adjacent to the rupture point for complete occlusion (Fig. 7).

Posttreatment DSA images showed complete resolution of the dCCF with aneurysm sac occlusion (Figs. 8A and 8B). 


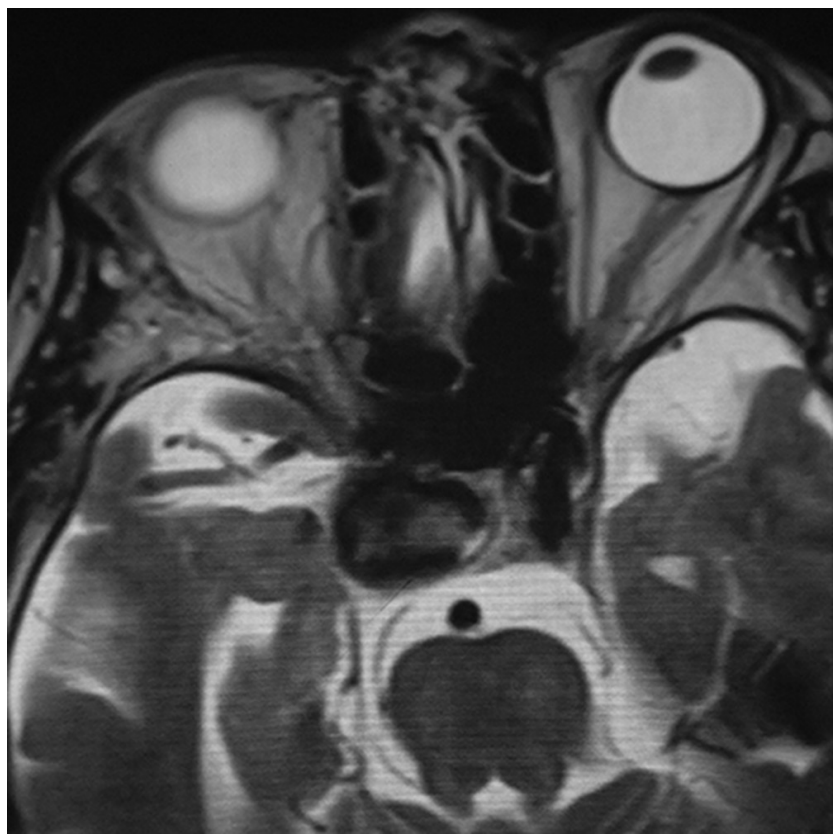

Fig. 1 Axial T2-weighted magnetic resonance image showing a right carotid cavernous aneurysm.

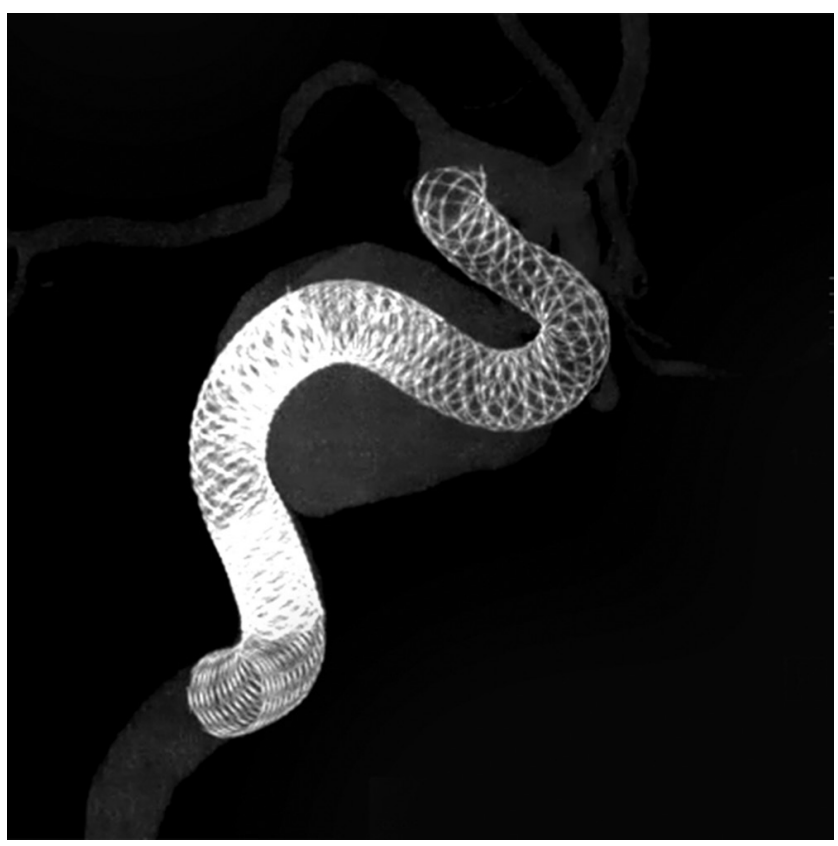

Fig. 3 Cone-beam computed tomography image showing three telescopically deployed PEDs within the aneurysm sac.

Pulsatile tinnitus disappeared and the right visual acuity gradually improved. The patient was discharged with the same DAPT regimen.

\section{Discussion}

Flow diverters disrupt the blood flow into the aneurysm sac and promote thrombosis while preserving the surrounding small vessels and perforators. PED is the most

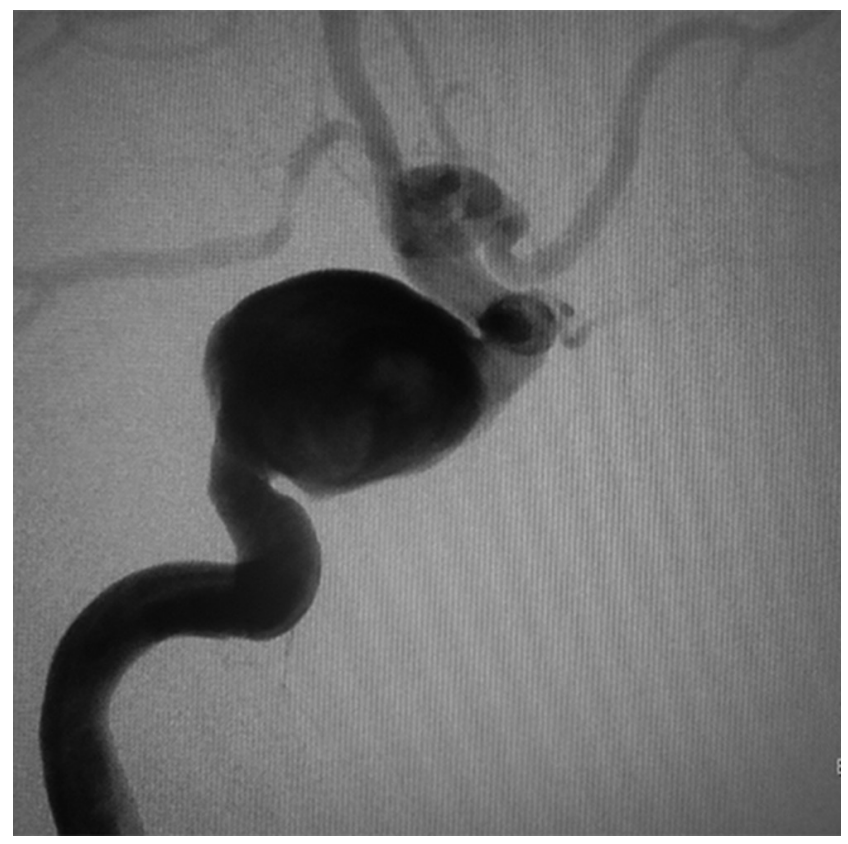

Fig. 2 Pre-treatment angiogram of the right ICA showing a 20-mm carotid cavernous aneurysm.

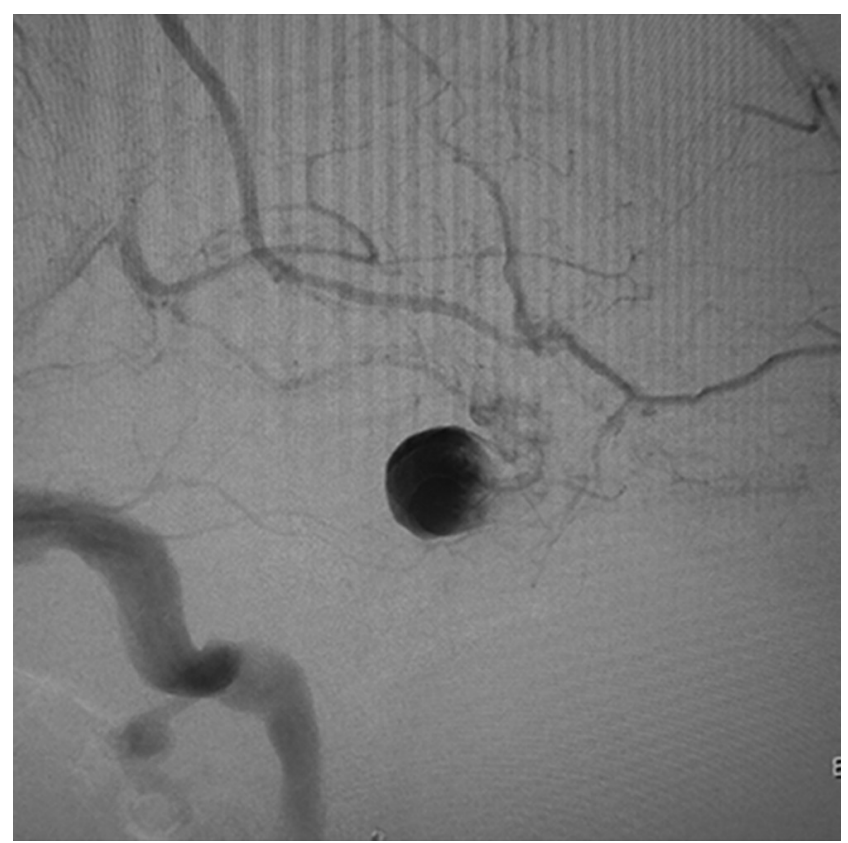

Fig. 4 Post-treatment angiogram of the right ICA showing significant stasis of contrast medium in the aneurysm sac.

common flow diverter which has recently been approved in Japan for large/giant unruptured or chronic ruptured aneurysms arising between the petrous and the superior hypophyseal artery segments of the ICA. Because the PED embolization is a less invasive procedure that does not involve a craniotomy, it has been increasingly used by incorporating the patients' wishes. Although some reports have shown the effectiveness and safety, ${ }^{4,7,8,9,10)}$ the delayed aneurysm rupture 

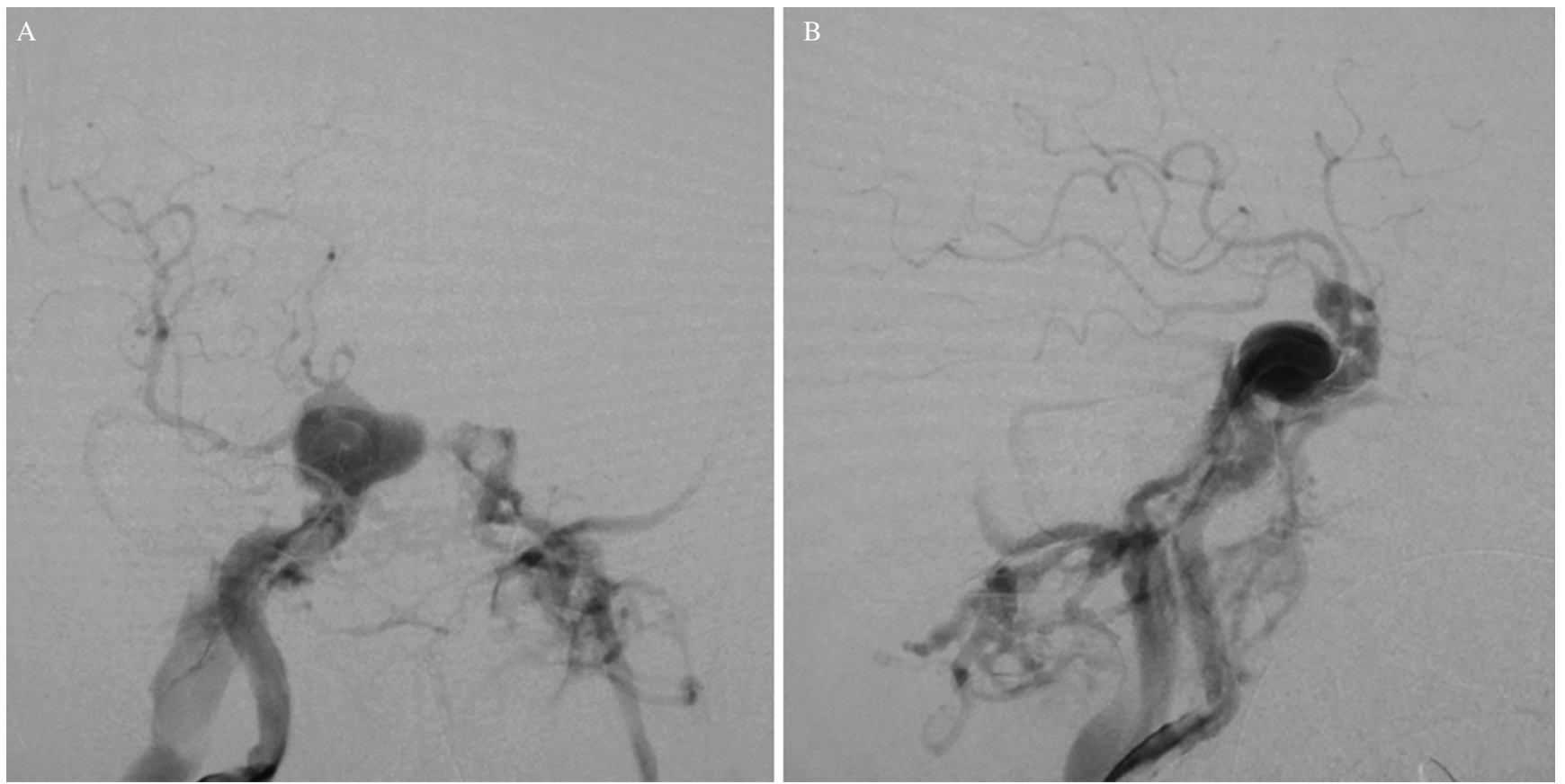

Fig. 5 Angiogram of the right ICA showing a dCCF: (A) frontal view, (B) lateral view.

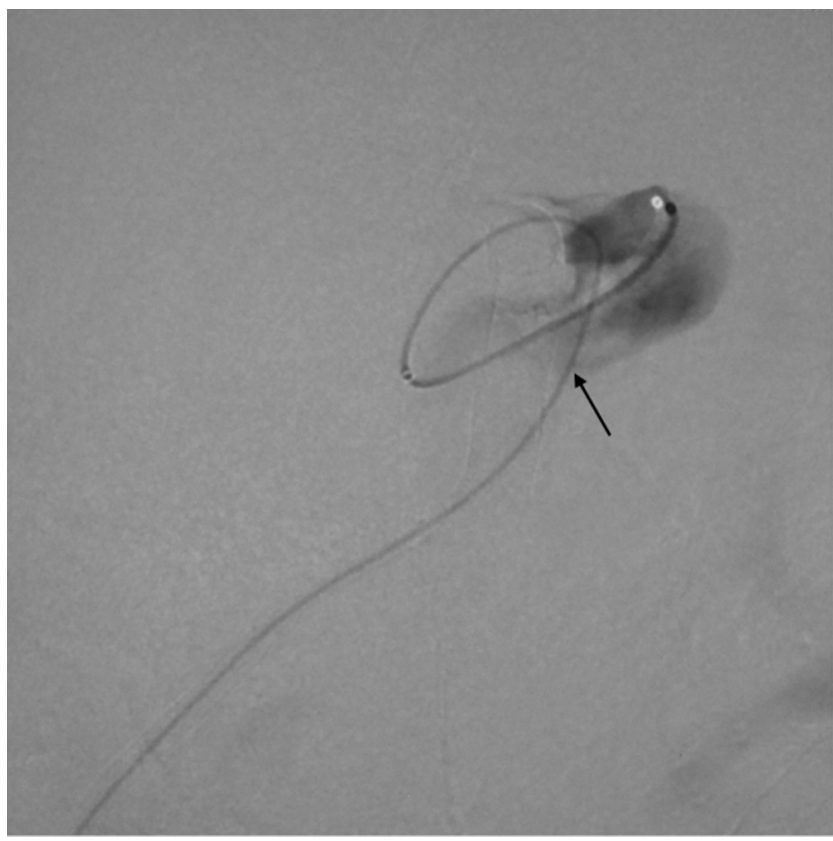

Fig. 6 A microcatheter was transvenously inserted into the aneurysm sac via the rupture point (arrow).

after PED embolization has been known as a rare but serious complication. ${ }^{1-6)}$ The etiology of a delayed aneurysm rupture is unclear, but the fragile aneurysm wall in the setting of inflammation coupled with continuous arterial pressures is suspected. ${ }^{11-13)}$ Some authors suggested that symptomatic large or giant aneurysms with high aspect ratio sac and/or inertiadriven inflow carry a high risk of delayed rupture after PED embolization. ${ }^{12-16)}$

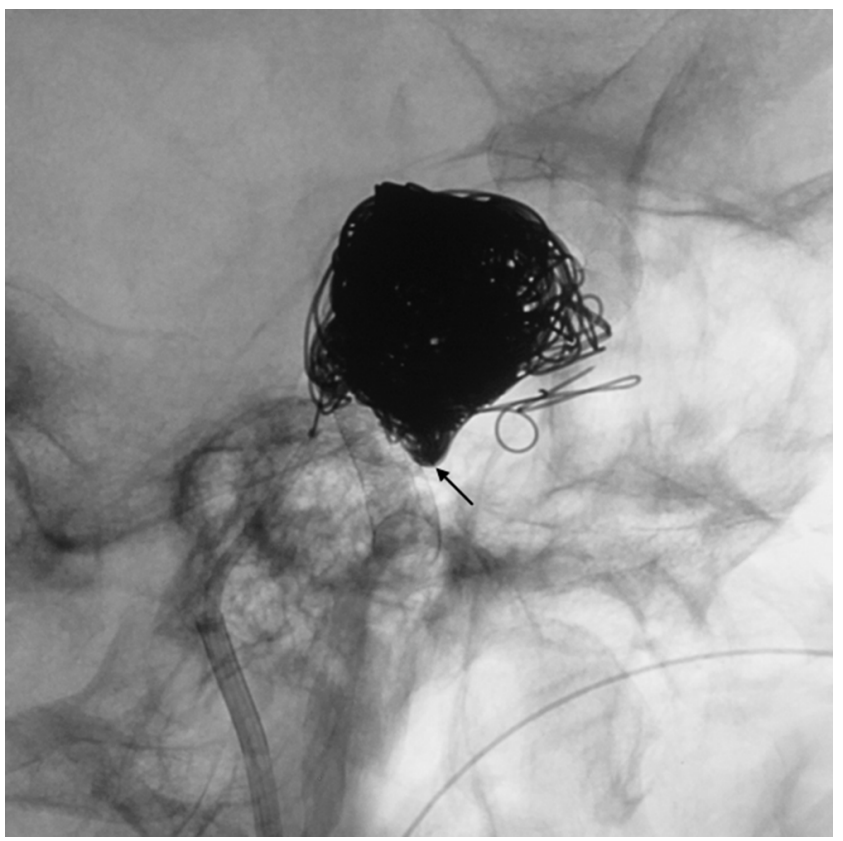

Fig. 7 Post-treatment lateral native image showing coil mass within the aneurysm sac and a small amount of coil mass within the CS adjacent to the rupture point (arrow).

Due to the anatomical specificity, the delayed rupture of carotid cavernous aneurysms cause dCCFs. The ideal treatment of dCCFs is to occlude the rupture point while preserving the ICA flow. Although the transarterial or transvenous coil embolization of the affected CS has become a primary treatment, the transarterial approach is not feasible because the meticulous mesh of flow diverters prohibits the microcatheter passage. But transvenous embolization associated with 

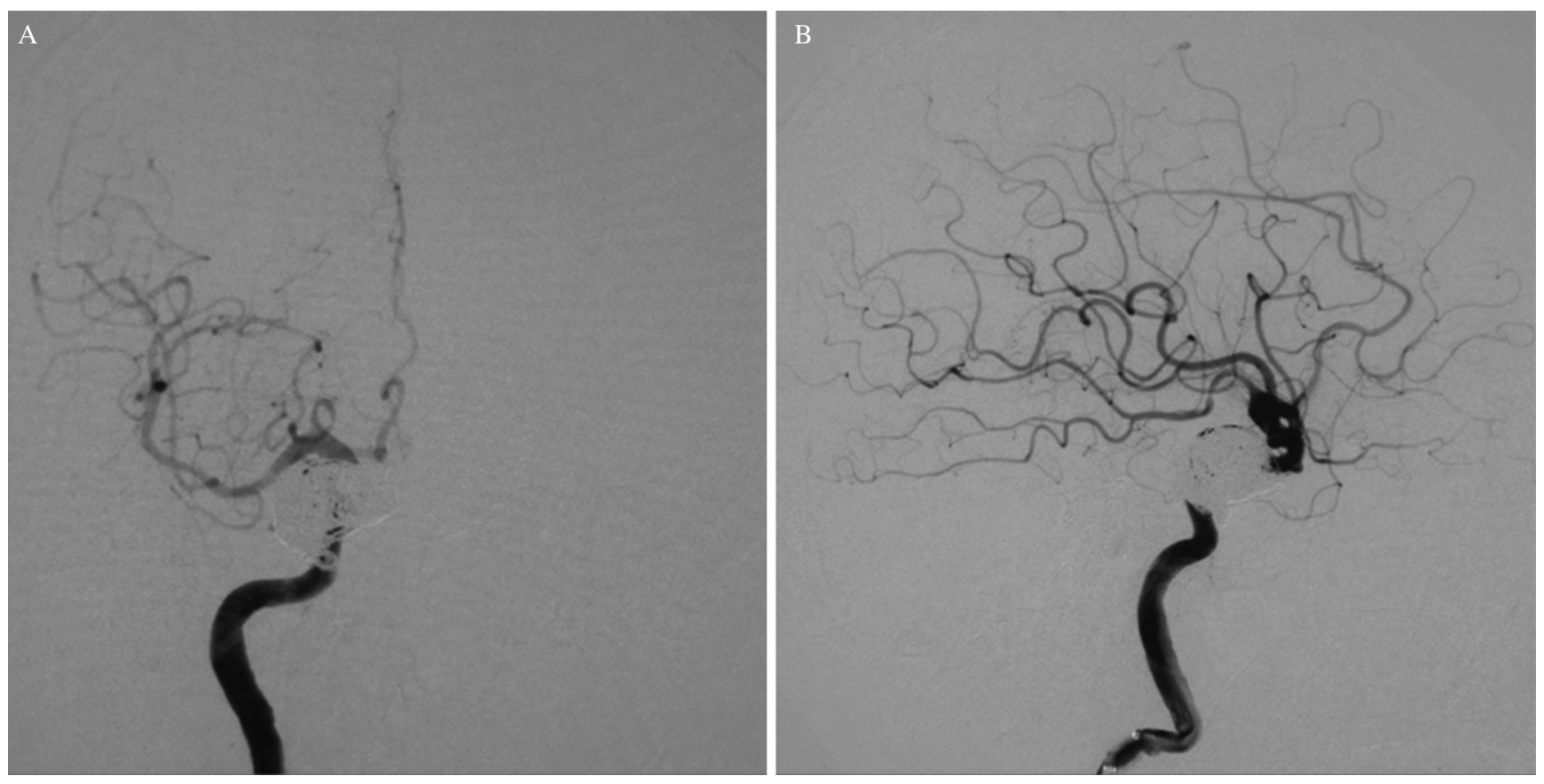

Fig. 8 Post-treatment angiograms of the right ICA showing complete disappearance of the dCCF: (A) frontal view, (B) lateral view.

Table 1 Summary of dCCF cases after flow diverter embolization

\begin{tabular}{|c|c|c|c|c|c|c|c|}
\hline Author (year) & Age & Gender & $\begin{array}{l}\text { Maximum aneurysm } \\
\text { size }(\mathrm{mm})\end{array}$ & $\begin{array}{l}\text { Interval to } \\
\text { rupture }\end{array}$ & Treatment & Access venous route & FD \\
\hline Mustafa (2010) & 39 & $\mathrm{~F}$ & 17.6 & 2 weeks & TVE & Facial vein/ophthalmic vein & Silk \\
\hline Kulcsar (2011) & 74 & F & 20 & 3 days & PAO & NA & Silk \\
\hline Kulcsar (2011) & 48 & F & 24 & 110 days & $\mathrm{PAO}$ & NA & Silk \\
\hline Lin (2015) & Middle age & NA & 10 & $<5$ weeks & TVE & Ipsilateral inferior petrosal sinus & Pipeline \\
\hline Lin (2015) & Middle age & NA & 17 & 3 days & TVE & Contralateral inferior petrosal sinus & Pipeline \\
\hline Ray (2016) & NA & NA & 17.4 & 11 days & $\mathrm{PAO}$ & NA & Pipeline \\
\hline Ray (2016) & NA & NA & 14.5 & 11 days & $\mathrm{PAO}$ & NA & Pipeline \\
\hline Ray (2016) & NA & NA & 31 & 3 days & $\mathrm{PAO}$ & NA & Pipeline \\
\hline Ray (2016) & NA & NA & 19 & 6 days & PAO & NA & Pipeline \\
\hline Ray (2016) & NA & NA & 18.8 & 7 days & $\mathrm{PAO}$ & NA & Pipeline \\
\hline Present case & 86 & $\mathrm{~F}$ & 20 & 6 weeks & TVE & Ipsilateral inferior petrosal sinus & Pipeline \\
\hline
\end{tabular}

dCCF: direct carotid cavernous fistula; FD: flow diverter; F: female; TVE: transvenous emoblization; PAO: parent artery occlusion; NA: not available.

the voluminous coil mass in the affected CS also carries the risk of permanent cranial nerve palsy in the CS. When the occlusion of the fistula while preserving the ICA flow is not possible, the BOT should be performed for the prediction of tolerance of a required permanent artery occlusion (PAO). Patients who can tolerate a PAO are indicated for the internal trapping of the ICA, but those who cannot require vascular revascularization because of the high risk of ischemia. But DAPT associated with flow diverter therapy is a possible cause of difficult hemostasis during vascular revascularization. Although the deployment of additional flow diverters was considered, there is only one case report so far. In the report, the occlusion of $\mathrm{dCCF}$ did not occur just after the procedure and confirmed at 1- year follow-up angiography. ${ }^{17)}$

To our knowledge, there are only four reports involving 10 patients with $\mathrm{dCCF}$ due to a delayed aneurysm rupture after flow diverter therapy (Table 1). ${ }^{13,18-20)}$ The flow diverters used were three Silk flow diverter stents (Balt, Extrusion, Montmorency, France) and seven PEDs. Seven patients were treated with PAO and the remaining 3 patients with transvenous coil embolization of the affected CS. ${ }^{3,18,19)}$

In the present case, the microcatheter was inserted transvenously into the aneurysm sac through the rupture point. Coil embolization of both the aneurysm sac and a part of the $\mathrm{CS}$ adjacent to the rupture point could achieve not only the 
immediate aneurysm occlusion but also the rupture point obliteration with a small amount of coil mass in the CS for preventing the newly developed or aggravated cranial nerve palsy due to the dense coil packing. ${ }^{21)}$ Finally, because the microcatheter insertion into the aneurysm sac via the rupture point is not always successful, the conventional transvenous embolization of CS with the disconnection of venous out flow should be considered.

\section{Conclusion}

PED embolization of carotid cavernous aneurysms may cause dCCFs due to a delayed aneurysm rupture. Transvenous aneurysm sac and rupture point coil embolization may be feasible and an ideal treatment option for immediate aneurysm occlusion and rupture point obliteration with a small amount of coil mass.

\section{Conflicts of Interest Disclosure}

The first author has been paid 1 million yen or more annually from Medtronic Japan for the time and effort required for attending conferences (presentations) and 10 million yen annually from Medtronic Japan as a research fund. There are no conflict of interests or financial interest that compromise the all co-authors' medical approach to patient management such as the materials and the devices in the article. All authors have registered online self-reported COI disclosure statement forms through the website for the Japan Neurosurgical Society.

\section{References}

1) Fischer S, Vajda Z, Aguilar Perez M, et al.: Pipeline embolization device (PED) for neurovascular reconstruction: initial experience in the treatment of 101 intracranial aneurysms and dissections. Neuroradiology 54: 369-382, 2012

2) Nelson PK, Lylyk P, Szikora I, Wetzel SG, Wanke I, Fiorella D: The pipeline embolization device for the intracranial treatment of aneurysms trial. AJNR Am J Neuroradiol 32: 34-40, 2011

3) Saatci I, Yavuz K, Ozer C, Geyik S, Cekirge HS: Treatment of intracranial aneurysms using the pipeline flow-diverter embolization device: a single-center experience with long-term follow-up results. AJNR Am J Neuroradiol 33: 1436-1446, 2012

4) Becske T, Kallmes DF, Saatci I, et al.: Pipeline for uncoilable or failed aneurysms: results from a multicenter clinical trial. Radiology 267: 858-868, 2013

5) McAuliffe W, Wycoco V, Rice H, Phatouros C, Singh TJ, Wenderoth J: Immediate and midterm results following treatment of unruptured intracranial aneurysms with the pipeline embolization device. AJNR Am J Neuroradiol 33: 164-170, 2012
6) Turowski B, Macht S, Kulcsár Z, Hänggi D, Stummer W: Early fatal hemorrhage after endovascular cerebral aneurysm treatment with a flow diverter (SILK-Stent): do we need to rethink our concepts? Neuroradiology 53: 37-41, 2011

7) Becske T, Brinjikji W, Potts MB, et al.: Long-term clinical and angiographic outcomes following pipeline embolization device treatment of complex internal carotid artery aneurysms: five-year results of the pipeline for uncoilable or failed aneurysms trial. Neurosurgery 80 : 40-48, 2017

8) Becske T, Potts MB, Shapiro M, et al.: Pipeline for uncoilable or failed aneurysms: 3-year follow-up results. J Neurosurg 127: 81-88, 2017

9) Kallmes DF, Brinjikji W, Cekirge S, et al.: Safety and efficacy of the pipeline embolization device for treatment of intracranial aneurysms: a pooled analysis of 3 large studies. J Neurosurg 127: 775-780, 2017

10) Kallmes DF, Brinjikji W, Boccardi E, et al.: Aneurysm study of pipeline in an observational registry (ASPIRe). Interv Neurol 5: 89-99, 2016

11) Ikeda H, Ishii A, Kikuchi $T$, et al.: Delayed aneurysm rupture due to residual blood flow at the inflow zone of the intracranial paraclinoid internal carotid aneurysm treated with the pipeline embolization device: histopathological investigation. Interv Neuroradiol 21: 674-683, 2015

12) Brinjikji W, Murad MH, Lanzino G, Cloft HJ, Kallmes DF: Endovascular treatment of intracranial aneurysms with flow diverters: a metaanalysis. Stroke 44: 442-447, 2013

13) Kulcsár Z, Houdart E, Bonafé A, et al.: Intra-aneurysmal thrombosis as a possible cause of delayed aneurysm rupture after flow-diversion treatment. AJNR Am J Neuroradiol 32: 20-25, 2011

14) Rouchaud A, Brinjikji W, Lanzino G, Cloft HJ, Kadirvel R, Kallmes DF: Delayed hemorrhagic complications after flow diversion for intracranial aneurysms: a literature overview. Neuroradiology 58: 171-177, 2016

15) Berge J, Biondi A, Machi P, et al.: Flow-diverter silk stent for the treatment of intracranial aneurysms: 1-year follow-up in a multicenter study. AJNR Am J Neuroradiol 33: 1150-1155, 2012

16) Kallmes DF, Hanel R, Lopes D, et al.: International retrospective study of the pipeline embolization device: a multicenter aneurysm treatment study. AJNR Am J Neuroradiol 36: 108-115, 2015

17) Amuluru K, Al-Mufti F, Gandhi CD, Prestigiacomo CJ, Singh IP: Direct carotid-cavernous fistula: a complication of, and treatment with, flow diversion. Interv Neuroradiol 22: 569-576, 2016

18) Lin LM, Colby GP, Jiang B, Pero G, Boccardi E, Coon AL: Transvenous approach for the treatment of direct carotid cavernous fistula following pipeline embolization of cavernous carotid aneurysm: a report of two cases and review of the literature. J Neurointerv Surg 7: e30, 2015

19) Mustafa W, Kadziolka K, Anxionnat R, Pierot L: Direct carotidcavernous fistula following intracavernous carotid aneurysm treatment with a flow-diverter stent. A case report. Interv Neuroradiol 16: 447-450, 2010

20) Roy AK, Grossberg JA, Osbun JW, et al.: Carotid cavernous fistula after pipeline placement: a single-center experience and review of the literature. J Neurointerv Surg 9: 152-158, 2017

21) Nishino $\mathrm{K}$, Ito $\mathrm{Y}$, Hasegawa $\mathrm{H}$, et al.: Cranial nerve palsy following transvenous embolization for a cavernous sinus dural arteriovenous fistula: association with the volume and location of detachable coils. J Neurosurg 109: 208-214, 2008

Corresponding author:

Hidenori Oishi, MD, PhD, Department of Neuroendovascular Therapy, Juntendo University School of Medicine, 2-1-1 Hongo, Bunkyo-ku, Tokyo 113-8421, Japan.

$\triangle$ ohishi@juntendo.ac.jp 